\title{
Diameter Effect of Silver Nanorod Arrays to Surface-enhanced Raman Scattering ${ }^{\dagger}$
}

\author{
Geun Hoi Gu, Min Young Kim, Hyeok Jin Yoon, and Jung Sang Suh* \\ Nano-materials Lab., Department of Chemistry, Seoul National University, Seoul 151-747, Korea \\ *E-mail: jssuh@snu.ac.kr \\ Received May 30, 2013, Accepted May 31, 2013
}

\begin{abstract}
The effect the diameter of silver nanorod arrays whose distance between the nanorods was uniform at $65 \mathrm{~nm}$ have on Surface-enhanced Raman Scattering (SERS) has been studied by varying the diameter from 28 to 51 $\mathrm{nm}$. Nanorod length was fixed at approximately $62 \mathrm{~nm}$, which is the optimum length for SERS by excitation with a $632.8 \mathrm{~nm}$ laser line. The transverse and longitudinal modes of the surface plasmon of these silver nanorods were near 400 and $630 \mathrm{~nm}$, respectively. The extinction of the longitudinal mode increased with increasing nanorod diameter, while the transverse mode did not change significantly. High-quality SERS spectra of $p$-aminothiophenol and benzenethiol adsorbed on the tips of the silver nanorods were observed by excitation with a $632.8 \mathrm{~nm}$ laser line. The SERS enhancement increased with increasing nanorod diameter. We concluded that the SERS enhancement increases when the diameter of silver nanorods is increased mainly by increasing the excitation efficiency of the longitudinal mode. The enhancement factor for the silver nanorods with a $51 \mathrm{~nm}$ diameter was approximately $2 \times 10^{7}$.
\end{abstract}

Key Words : Surface-enhanced Raman scattering, SERS, Silver nanorod arrays

\section{Introduction}

Surface-enhanced Raman Scattering (SERS) is emerging as a probing technique for biosensors due to its high sensitivity. ${ }^{1-8}$ It is known that SERS has the sensitivity to detect even single molecules. ${ }^{9-14}$ For such high-sensitivity detection, a very high surface enhancement - up to $10^{11}$ - may be required. ${ }^{15}$ It is known that a very strong enhancement is obtained from particular sites, the so-called hot spots, which may be junctions between nanoparticles. ${ }^{16-19}$ However, it is not easy to make a junction system intentionally by injecting nanoparticles to a system, especially to a bio-system. For practical application of SERS, a simple system rather than a junction system should be used. It would be ideal if single nanoparticles have enough enhancements to detect single molecules. For this, it is important to maximize the enhancement of single nanoparticles.

Silver nanowire arrays fabricated on anodic aluminum oxide (AAO) templates have been used as a substrate for SERS. ${ }^{16,20-25}$ In the fabrication of nanowires using AAO templates, the diameter and length of the nanowires can be controlled easily. The length can be controlled by controlling the deposition time, and the diameter can be adjusted by depositing silver in the pores of an AAO template whose diameter has been controlled by a pore widening technique. The optical property of silver nanowires depends on their diameter and length. ${ }^{26-28}$ When the nanowires are relatively short, two absorption bands appear due to the transverse and longitudinal modes of the dipolar plasmon. The transverse mode is parallel to the short axis of the wires and its absorp-

This paper is to commemorate Professor Myung Soo Kim's honourable retirement. tion band appears in the short wavelength region; on the other hand, the longitudinal mode is parallel to the long axis and its plasmon wavelength is shifted toward red with increasing length of the nanowires. When the length becomes large, the absorption band of the longitudinal mode of the dipolar plasmon is shifted close to the infrared region and the absorption bands due to multipolar plasmons appearing in the visible region. ${ }^{29}$ Therefore, the longitudinal mode of the dipolar plasmon of long nanowires undergoes minimal excitation by irradiation with a visible laser line. Recently, we have found that the optimum length of silver nanowires for SERS is very short. ${ }^{25,30}$ It is approximately $62 \mathrm{~nm}$ for excitation with a $632.8 \mathrm{~nm}$ laser line. For silver nanorods with a diameter of $31 \mathrm{~nm}$ and $62 \mathrm{~nm}$ in length, the transverse mode of the surface plasmon is near $400 \mathrm{~nm}$, while the longitudinal mode is near $630 \mathrm{~nm}$. The longitudinal mode is very close to the excitation laser line, while the transverse mode is far from it. By excitation with a $632.8 \mathrm{~nm}$ laser line, the SERS of molecules adsorbed on the tip of silver nanorods fabricated on an AAO template is mainly contributed by exciting of the longitudinal mode of the surface plasmon. In the previously reported paper, ${ }^{25,30}$ only the length of silver nanorods was optimized for the excitation laser line; the diameter was not optimized simultaneously. In this paper, we have studied the effect the diameter of silver nanorod arrays have on SERS while the length of the silver nanorods is optimized to the excitation laser line.

\section{Experimental}

A highly ordered porous AAO template was fabricated by using a two-step anodization technique. ${ }^{31}$ Clean aluminum sheets $(99.999 \%, 0.5 \mathrm{~mm}$ thickness, Good fellow Ltd.) were 
anodized in $0.5 \mathrm{M}$ sulfuric acid at $10^{\circ} \mathrm{C}$ and at a constant applied voltage of $25 \mathrm{~V}$ for $24 \mathrm{~h}$ after annealing and electropolishing. The resultant aluminum oxide film was subsequently removed by dipping the anodized sheet into an aqueous mixture of chromic acid (1.8 wt \%) and phosphoric acid $(6 \mathrm{wt} \%)$ at $60^{\circ} \mathrm{C}$ for $16 \mathrm{~h}$. The second anodization was performed for $5 \mathrm{~min}$ under the same conditions as the first. The thickness of the AAO film was approximately $800 \mathrm{~nm}$. The pore diameter was widened by etching in a $0.1 \mathrm{M}$ phosphoric acid solution at $30^{\circ} \mathrm{C}$ for 15 to $30 \mathrm{~min}$. In this case, the bottom barrier layer was also reduced. Silver nanorods were deposited in the widened pores of the AAO templates by applying an AC voltage of 13-17 V with a frequency of $200 \mathrm{~Hz}$ in ethanol solution containing $0.05 \mathrm{M}$ $\mathrm{AgNO}_{3}\left(99.9999 \%\right.$, Aldrich) at $5{ }^{\circ} \mathrm{C}$ (see Fig. 1). Each of the AAO templates deposited with silver nanorods was dipped into separate $1.0 \times 10^{-3} \mathrm{M} p$-aminothiophenol or benzenethiol solutions for $30 \mathrm{~min}$ and then their surfaces were washed with ethanol. After washing, oxide of the AAO templates were partially etched away to expose the silver nanorods deposited. This was done by dipping each template into separate $0.1 \mathrm{M}$ phosphoric acid solutions at $30^{\circ} \mathrm{C}$ for varying times (from 15 to $35 \mathrm{~min}$ ) and then dried for Raman measurements after washing the surface with ethanol. Raman spectra were observed by using a micro-Raman system equipped with a homemade sample stage, a monochrometer (SPEX $500 \mathrm{M}$ ), and a CCD camera cooled with liquid nitrogen (Roger Scientific 7346-001 Model). The incident laser power on the sample was approximately $100 \mu \mathrm{W}$, and the acquisition time was $1 \mathrm{~s}$. The UV-visible extinction spectrum of the silver nanorod arrays fabricated on an AAO template was measured by a diffuse reflection method using a Cary Varian 300 Bio/diffuse-reflectance kit. The fabricated templates and nanorods were analyzed by using a scanning electron microscope (SEM; Philips FEG XL (30 kV)).

\section{Results and Discussion}

The top SEM images of the silver nanorods deposited in the pores of the AAO template prepared in sulfuric acid, after partially exposing by etching away of some oxide, are shown in Figure 1. Silver nanorods are deposited in all the pores. The surfaces are relatively unclean. This may be due to the fact that the oxide has not been etched away evenly. Also, some cracks have been formed during oxide etching. Silver nanorods exhibit a two-dimensional array with a hexagonal pattern. The distance between the center of one nanorod and the centers of the surrounding nanorods is uniform at $65 \mathrm{~nm}$. The nanorod density is approximately $2.7 \times 10^{10}$ nanorods $/ \mathrm{cm}^{2}$. The diameters of the nanorods are uniform. The diameter of the nanorods is approximately (a) 28, (b) 34 , (c) 43 , and (d) $51 \mathrm{~nm}$. The length of all the nanorods is the same at approximately $62 \mathrm{~nm}$, which is the optimum length for SERS in excitation caused by a 632.8 $\mathrm{nm}$ laser line. ${ }^{25}$ The pore depth of the AAO templates was approximately $800 \mathrm{~nm}$. This was much greater than the length of the deposited silver nanorods. After adsorption of
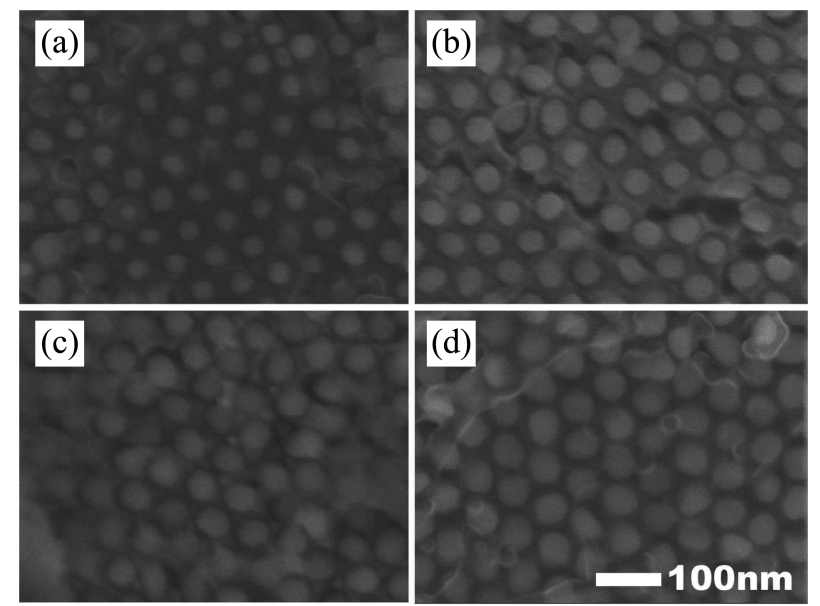

Figure 1. The SEM images of the Ag nanorods electrodeposited in the pores of AAO templates, whose pores were widened for 15 to $30 \mathrm{~min}$, after etching away some oxide. The diameter of silver nanorods is approximately (a) 28, (b) 34 , (c) 43 and (d) $51 \mathrm{~nm}$. The length of all the nanorods is the same at approximately $62 \mathrm{~nm}$.

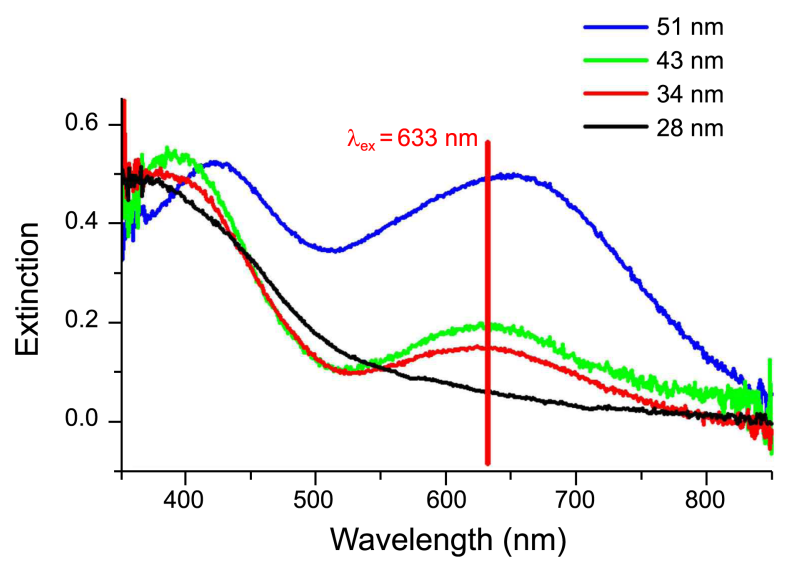

Figure 2. The UV-visible extinction spectra of the Ag nanorod arrays, measured by a reflection method. The legend shows the diameters of the silver nanorods. The length of all the nanorods was the same at approximately $62 \mathrm{~nm}$.

molecules, oxide of the AAO templates was partially etched away to expose the silver nanorods because without etching, the SERS signal was relatively weak due to the interference of oxide.

The UV-visible extinction spectra of the silver nanorod arrays fabricated on AAO templates, measured by a diffuse reflection method, are shown in Figure 2. In each spectrum, there are two bands near 400 and $633 \mathrm{~nm}$. Since our silver nanorods are very short at $62 \mathrm{~nm}$, these bands could be assigned simply: the former is due to the transverse mode of the surface plasmon and the latter is due to the longitudinal mode..$^{32}$ The transverse mode is slightly red-shifted from approximately 390 to $430 \mathrm{~nm}$ when increasing the diameter of silver nanorods from 28 to $51 \mathrm{~nm}$. For example, it is near $430 \mathrm{~nm}$ for the nanorods with a $51 \mathrm{~nm}$ diameter, while near $390 \mathrm{~nm}$ for a $34 \mathrm{~nm}$ diameter. However, the values of extinction are very similar. The maximum position of the longitudinal mode is very close to the $632.8 \mathrm{~nm}$ laser line of 


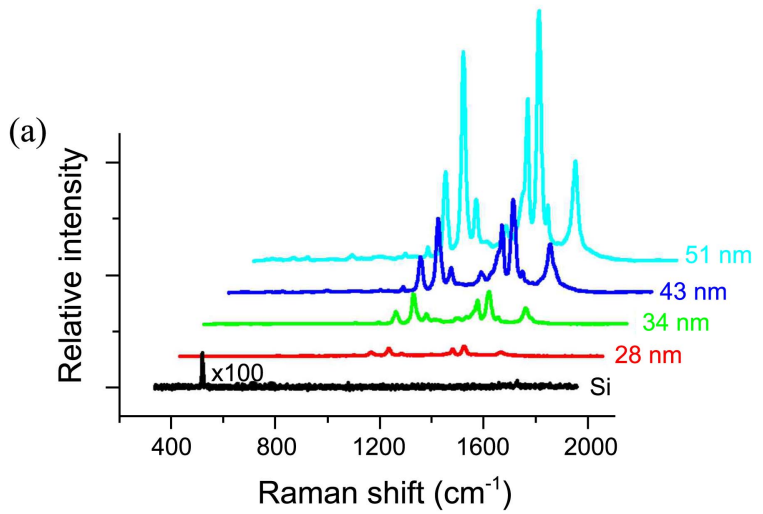

(b)

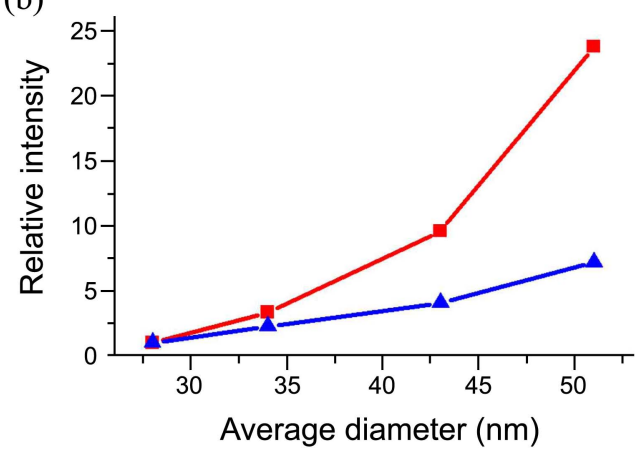

Figure 3. (a) The SERS spectra of $p$-aminothiophenol adsorbed at the tip of silver nanorods fabricated on AAO templates, observed by excitation with a $632.8 \mathrm{~nm}$ laser line and using a highmagnification objective lens $(50 \times)$. (b) The relative intensities of the peaks at $1077 \mathrm{~cm}^{-1}$ in the SERS spectra shown in (a): before (squares) and after (triangles) correcting the factor of the diameter of silver nanorods. The value labeled at the right-hand side in (a) is the diameter of the silver nanorods. The length of all the nanorods was the same at approximately $62 \mathrm{~nm}$.

a helium-neon laser. The extinction increases significantly with increasing diameter. The maximum position of the longitudinal mode does not change very much with increasing diameter. This may mean that the optimum length of silver nanorods for SERS does not change significantly with a change in diameter.

The SERS spectra of $p$-aminothiophenol adsorbed at the tip of silver nanorod arrays are shown in Figure 3. They were measured using a micro-Raman system by excitation with a $632.8 \mathrm{~nm}$ laser line and in the usual way by laser beam irradiation perpendicular to the surface of the sample template placed on the sample stage. The SERS peaks correspond to the modes of a benzene ring. ${ }^{33}$ For example, the peak at $1573 \mathrm{~cm}^{-1}$ is due to $8 \mathbf{b}$ and the one at $1440 \mathrm{~cm}^{-1}$ is due to $19 \mathbf{b}$. Moreover, the peak at $1077 \mathrm{~cm}^{-1}$ is due to $7 \mathbf{a}$ with contributions from the C-S stretching vibration (CS). The SERS intensity increases when increasing the diameter of silver nanorods. For the peak at $1077 \mathrm{~cm}^{-1}$, the intensity is approximately 650, 2300, 6700 and 16000 for the nanorods of $28,34,43$ and $51 \mathrm{~nm}$ in diameter, respectively. The relative intensity is $1: 3.5: 10.3: 24.6$. However, the number of molecules measured was not the same. The molecules can be adsorbed only on the tip of the nanorods, since the nanorods are deposited in the pores of AAO templates and only the tip is exposed. It should be mentioned that oxide etching was done after adsorption. Therefore, the number of molecules adsorbed on the tips is proportional to the tip surface area, which is proportional to the square of the diameter. Here, we have assumed a monolayer adsorption, since we had washed the surface thoroughly following a relatively long adsorption. Since the diameters were 28,34 , 43 , and $51 \mathrm{~nm}$, the relative tip surface area of these nanorods was 1:1.47:2.36:3.32. After correcting the factor of different tip surface areas, the relative intensity becomes 1:2.4:4.4: 7.4. For all the samples, the number density of silver nanorods and the length were the same. The only difference was the diameter of the silver nanorods. However, even after dividing by the factor of different number of molecules adsorbed on the tips, the relative SERS intensity still increases with increasing diameter. Therefore, it is concluded that the SERS enhancement on silver nanorod arrays increases with increasing nanorod diameter. For our silver nanorod arrays, the distance between the center of one nanorod and the centers of the surrounding nanorods is uniform at $65 \mathrm{~nm}$. As the diameter increases, the gap distance between neighboring silver nanorods decreases and the gap-to-diameter ratio decreases. Therefore, our results could be expressed as such: the SERS enhancement on silver nanorod arrays increases with decreasing gap-to-diameter ratio. A similar behavior has been observed from silver nanowire arrays, whose length is about $50 \mu \mathrm{m} .{ }^{34}$ Also, a theoretical calculation for a similar system has been reported. ${ }^{35}$ In this calculation, the distance between hexagonally arranged hemispheres has been fixed and changed the particle size. In this case, the enhancement increases with increasing particle size.

In the UV-visible extinction spectra, there are two bands near 400 and $633 \mathrm{~nm}$ (see Figure 2). The transverse mode of the surface plasmon of silver nanorod arrays near $400 \mathrm{~nm}$ is slightly shifted from 390 to $430 \mathrm{~nm}$ when the diameter is increased from 28 to $51 \mathrm{~nm}$. No significant extinction change is observed. The wavelength of $430 \mathrm{~nm}$ is far apart from the excitation wavelength, $632.8 \mathrm{~nm}$. Therefore, the transverse mode could be hardly excited under this condition. For the longitudinal modes near $633 \mathrm{~nm}$, the wavelength shows a slight change but the extinction is significantly increased when nanorod diameter is increased. If the excitation efficiency of the longitudinal mode increases with increasing nanorod diameter, the extinction of the longitudinal mode will increase. When the extinction of the longitudinal mode of silver nanorods increases, the local electromagnetic field at or near silver nanorods will increase, and consequently the SERS enhancement will increase. We have previously concluded that the SERS enhancement on silver nanorod arrays increases with increasing diameter. Therefore, it is concluded that the SERS enhancement increases by increasing the extinction of the longitudinal mode of the surface plasmon of silver nanorod arrays. However, it is not clear that how the extinction of the longitudinal mode of silver nanorods increases with increasing diameter.

For our silver nanorod arrays, the length is fixed at $62 \mathrm{~nm}$ 
and the distance between the center of one nanorod and the centers of the surrounding nanorods is uniform at $65 \mathrm{~nm}$. Therefore, with increasing diameter, the volume of each nanorod increases and the surface-to-surface distance between neighboring nanorods decreases. We can consider two possible mechanisms for the extinction increase. One is that the extinction of each silver nanorod increases with increasing diameter. The volume for oscillation of the longitudinal mode in each silver nanorod would increase as diameter increases, since the length of silver nanorods was fixed at 62 $\mathrm{nm}$, which is the optimum length for SERS by excitation with a $632.8 \mathrm{~nm}$ laser line. Therefore, as nanorod diameter increases, the amplitude of the longitudinal mode could increase and the extinction or excitation efficiency of the longitudinal mode of each nanorod could increase. The other possible mechanism is that the surface-to-surface distance between neighboring silver nanorods decreased with increasing diameter and the extinction of the longitudinal mode of silver nanorods could be increased by the interaction between silver nanorods. When silver nanoparticles of a proper size are joined or located within high proximity, the enhancement increases compared to that of isolated nanoparticles. ${ }^{30}$ The enhancement increase is known as the junction effect. However, for our silver nanorods, the closest average distance was $14 \mathrm{~nm}$, since the inter nanorods distance was very uniform to $65 \mathrm{~nm}$ and the largest diameter was $51 \mathrm{~nm}$. Even the closest distance might be too far to achieve the junction effect. If the extinction of the longitudinal mode of silver nanorods was increased somewhat by the interaction between silver nanorods, it might be due to a long-range interaction between silver nanorods. Unfortunately, under our experimental conditions, the volume of silver nanorod and the surface-to-surface distance between neighboring silver nanorods were changed simultaneously with increasing diameter. Therefore, we could not isolate the measurement of only one effect. This should be studied further theoretically and experimentally.

For our silver nanorod arrays, the aspect ratio (length to diameter) decreases with increasing diameter, since the nanorod length is fixed at $62 \mathrm{~nm}$. We expect an increase in enhancement if we increase the aspect ratio via a so-called lighting rod effect. Although the aspect ratio decreases with diameter increase, our results show that the SERS enhancement on silver nanorods increases with increasing diameter when the length is fixed as the optimum length for SERS. The reason should be studied further theoretically and experimentally. The smallest aspect ratio of our silver nanorods fabricated is 1.2 , since the largest diameter is $51 \mathrm{~nm}$ and the corresponding length is the same at $62 \mathrm{~nm}$. We could not fabricate silver nanorods whose aspect ratio is smaller than 1.2 because our AAO templates whose inter-pore distance was $65 \mathrm{~nm}$ were ruined when the pore diameter was widened larger than $51 \mathrm{~nm}$. The bottoms of our silver nanorods have a hemispheric shape, while the tops do not have a hemispheric shape (see Figure 1). As we increased the diameter, the top of our silver nanorods became more flat. Therefore, if we could fabricate the silver nanorods with an aspect ratio of (a)

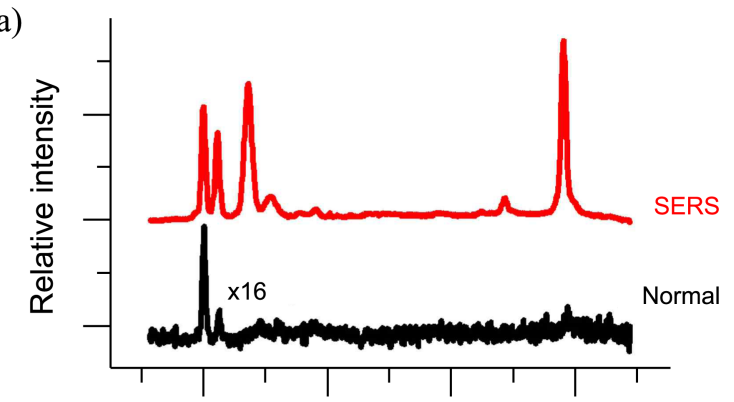

(b)

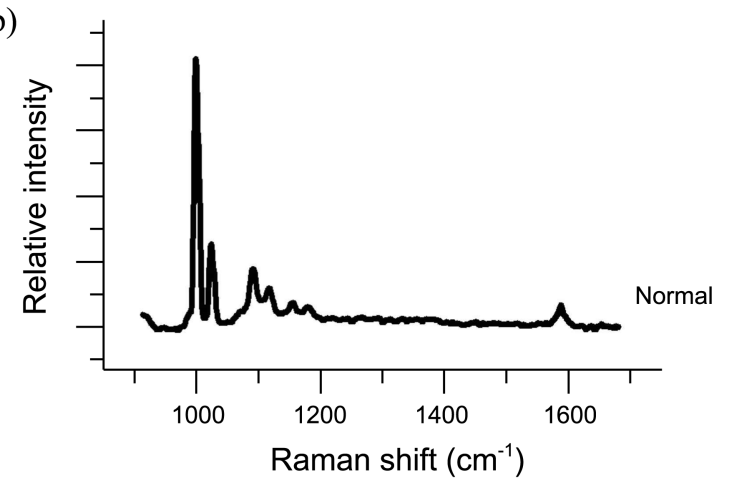

Figure 4. (a) Comparison of the normal Raman spectrum of pure benzenethiol liquid with its SERS spectrum adsorbed at the tip of the $51 \mathrm{~nm}$ silver nanorods in diameter and (b) the normal Raman spectrum of pure liquid accumulated for a long time; both the spectra in (a) were observed by excitation with a 623.8-nm laser line and using a low-magnification objective lens $(10 \times)$ under the same conditions. The normal Raman spectrum is magnified 16 times to normalize the intensity of the peak at $1000 \mathrm{~cm}^{-1}$.

approximately 1 , they may not have a spherical shape. To find the optimal shape or geometry for SERS, more experiments may be needed.

We calculated the enhancement factor by comparing the SERS intensity of benzenethiol with its normal Raman intensity of the peaks at $1000 \mathrm{~cm}^{-1}$ by using a technique similar to that reported previously. ${ }^{25}$ The normal Raman spectrum and the SERS spectrum of benzenethiol that were obtained by using a low-magnification objective lens $(10 \times)$ in an acquisition time of $100 \mathrm{~s}$-are shown in Figure 4. [The SERS spectrum is very similar to that reported previously. ${ }^{25}$ ] The beam diameter was approximately $5 \mu \mathrm{m}$. It should be mentioned that in the measurement of the enhancement factor, we used a $10 \times$ lens to observe the SERS and normal Raman spectra because with a high magnification lens, the laser beam does not assume a cylindrical form in the liquid sample. In such a case, it is difficult to estimate the number of molecules irradiated by the laser beam. The intensity of the SERS peak observed from the molecules adsorbed on silver nanorods of $51 \mathrm{~nm}$ in diameter is very similar to that of the normal Raman peak magnified 16 times. The individual cross-sectional area of benezenethiol is $0.147 \mathrm{~nm}^{2} .^{36}$ Assuming that the molecules were adsorbed as a monolayer at the tip of each nanorod with a diameter of $\sim 51 \mathrm{~nm}$ whose surface area is $4.08 \times 10^{3} \mathrm{~nm}^{2}$ by assuming a half sphere $\left[4 \times 3.14 \times(25.5 \mathrm{~nm})^{2} / 2=4.08 \times 10^{3} \mathrm{~nm}^{2}\right]$, approximately 
$2.78 \times 10^{4}$ molecules were adsorbed on the tip of each silver nanorod, $\left[4.08 \times 10^{3} \mathrm{~nm}^{2} /\left(0.147 \mathrm{~nm}^{2}\right)=2.78 \times 10^{4}\right]$. Since the surface area irradiated by a $5 \mu \mathrm{m}$-diameter laser beam is $19.6 \mu \mathrm{m}^{2},\left[3.14 \times(2.5 \mu \mathrm{m})^{2}=19.6 \mu \mathrm{m}^{2}\right]$, and the nanorod density is approximately $2.7 \times 10^{10}$ nanorods $/ \mathrm{cm}^{2}, 5.29 \times 10^{3}$ silver nanorods exist in the laser beam spot, [19.6 $\mu^{2} \times$ $2.7 \times 10^{10}$ nanorods $/ \mathrm{cm}^{2}=5.29 \times 10^{3}$ nanorods]. Therefore, approximately $1.47 \times 10^{8}$ molecules were sampled by the laser beam with a beam diameter of $5 \mathrm{~m},\left[\left(2.78 \times 10^{4}\right.\right.$ molecules $/$ nanorod $) \times\left(5.29 \times 10^{3}\right.$ nanorods $)=1.47 \times 10^{8}$ molecules]. The normal Raman spectrum was observed for a 100 $\mathrm{m}$-thick cell filled with pure benzenethiol liquid that had a density of $1.08 \mathrm{~g} / \mathrm{cm}^{3}$. The molecular mass of benzenethiol is $110.18 \mathrm{~g} / \mathrm{mol}$. The probe volume was approximately $1962.5 \mu \mathrm{m}^{3}$, calculated by assuming that it is a cylinder with a diameter of $5.0 \mathrm{~m}$ and a height of $100 \mathrm{~m},\left[3.14 \times(2.5 \mathrm{~m})^{2}\right.$ $\left.\times 100 \mathrm{~m}=1962.5 \mu \mathrm{m}^{3}\right]$. Under these conditions, $1.16 \times 10^{13}$ molecules would be irradiated, $\left[1962.5 \mu \mathrm{m}^{3} \times 1.08 \mathrm{~g} / \mathrm{cm}^{3}\right.$ $\times(110.18 \mathrm{~g} / \mathrm{mol})^{-1} \times 6.02 \times 10^{23} \mathrm{molecules} / \mathrm{mol}=1.16 \times 10^{13}$ molecules]. From this data of the relative intensity and the number of molecules sampled from the normal Raman and SERS measurements, the enhancement factor is calculated to be approximately $1.26 \times 10^{6}$, $\left[16 \times\left(1.16 \times 10^{13}\right.\right.$ molecules $) /$ $\left(1.47 \times 10^{8}\right.$ molecules $\left.)=1.26 \times 10^{6}\right]$. It should be mentioned that in the normal Raman spectrum the peak at $1000 \mathrm{~cm}^{-1}$ is the strongest and the peak at $1573 \mathrm{~cm}^{-1}$ is relatively weak, while in the SERS spectrum the peak at $1573 \mathrm{~cm}^{-1}$ is the strongest. In the normal spectrum, the peak at $1000 \mathrm{~cm}^{-1}$ is approximately 12 times stronger than the peak at $1573 \mathrm{~cm}^{-1}$ (see Figure 4(b)). In the SERS spectrum, the peak at 1573 $\mathrm{cm}^{-1}$ is approximately 1.5 times stronger than that at 1000 $\mathrm{cm}^{-1}$. If we consider these factors, the enhancement factor calculated by comparing the relative intensities of the peaks at $1573 \mathrm{~cm}^{-1}$ will become $2.3 \times 10^{7}$.

We also calculated the enhancement factor using the data of $p$-aminothiophenol shown in Figure 5. The intensity of the SERS peak is very similar to that of the normal Raman

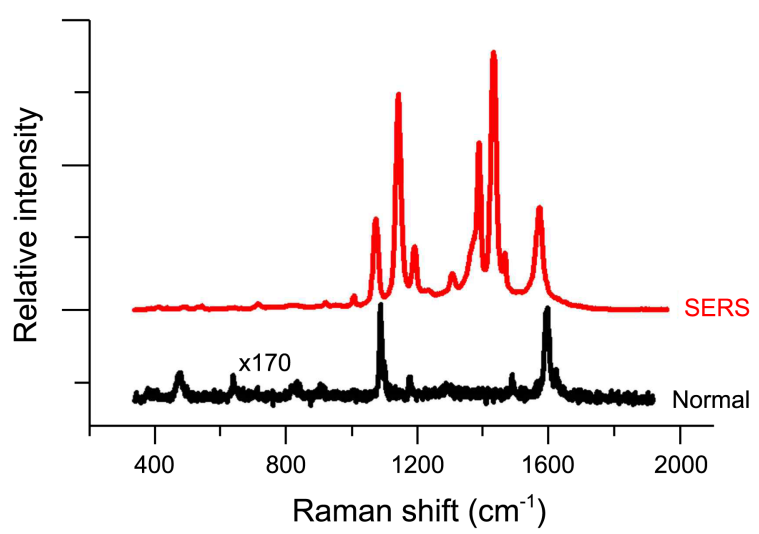

Figure 5. Comparison of the normal Raman spectrum of pure $p$ aminothiophenol liquid with its SERS spectrum adsorbed at the tip of the $51 \mathrm{~nm}$ silver nanorods in diameter; both the spectra were observed by excitation with a 623.8-nm laser line and using a lowmagnification objective lens $(10 \times)$ under the same conditions. The normal Raman spectrum is magnified 170 times to normalize the intensity of the peak at $1077 \mathrm{~cm}^{-1}$. peak magnified 170 times. The density of pure $p$-aminothiophenol liquid is $1.06 \mathrm{~g} / \mathrm{cm}^{3}$ and the individual cross-sectional area of $p$-aminothiophenol is $0.22 \mathrm{~nm}^{2} \cdot{ }^{37}$ By a similar calculation the enhancement factor is given as approximately $1.7 \times 10^{7}$. Based on the data of benzenetiol, the enhancement factor calculated is approximately $2.3 \times 10^{7}$. Therefore, it is concluded that the enhancement factor is approximately $2 \times 10^{7}$. The enhancement measured may be mainly due to electromagnetic field enhancement, since $p$-aminothiophenol and benzenethiol have no absorption in the visible and are nonresonant molecules. It should be mentioned that our calculated enhancement value may represent the average enhancement for all the tip area of silver nanorods, since the edge and middle of the tips may have a different enhancement. The normal Raman samples were transparent, since a cell filled with the liquid samples was used in the normal Raman measurements. However, the SERS samples were non-transparent substrates with silver nanorods. Therefore, the normal Raman signals could be excited only by the incident beam, while the SERS signals could be excited by both the incident and reflected beams. This means that there was an underestimation of the normal Raman signals. Consequently, the enhancement value of $2 \times 10^{7}$ could be somewhat overestimated. However, the factor may be smaller than 2, since the reflected beam could not be stronger than the incident beam.

The SERS spectra were measured using a micro-Raman system and in the usual way by laser beam irradiation perpendicular to the surface of the sample template placed on the sample stage. The alignment of the $\mathrm{Ag}$ nanorods is perfectly perpendicular with respect to the surface of the template. In this case, the polarization direction of the laser beam is parallel to the short axis of the nanorods, while being perpendicular to the long axis. In this geometry, the transverse mode of the surface plasmon can be excited by irradiation, while the longitudinal mode cannot. However, it can be shown that it is possible to excite the longitudinal mode under our experimental conditions through the following reasoning. For the micro-Raman system, a lens with a very short focal length was used simultaneously to focus the laser beam and to detect the Raman signals. In this case, the edge of the laser beam would be refracted through a wide angle. ${ }^{38}$ Part of the refracted laser beam would irradiate the nanorods at an angle. Therefore, the longitudinal mode of the surface plasmon of the nanorods can be excited, since the refracted laser beam has a polarization component parallel to the long axis of the nanorods. The evidence for this argument was discussed in detail in our previous paper. ${ }^{25}$

\section{Conclusion}

The effect the diameter of silver nanorod arrays whose distance between nanorods was uniform at $65 \mathrm{~nm}$ have on SERS has been studied by varying the diameter from 28 to $51 \mathrm{~nm}$, while their length was fixed at approximately $62 \mathrm{~nm}$, which is the optimum length for SERS by excitation with a $632.8 \mathrm{~nm}$ laser line. The SERS enhancement increased when 
the diameter was increased. The extinction of the longitudinal mode of the surface plasmon of silver nanorods near $630 \mathrm{~nm}$ increased with increasing diameter of silver nanorods, while that of the transverse mode near $400 \mathrm{~nm}$ was almost unchanged. It is concluded that the SERS enhancement increases with increasing diameter of silver nanorods by increasing the excitation efficiency of the longitudinal mode. The enhancement for silver nanorods with a $51 \mathrm{~nm}$ in diameter was approximately $2 \times 10^{7}$.

Acknowledgments. This research was supported by Basic Research Program through the National Research Foundation of Korea (NRF) funded by the Ministry of Education, Science and Technology (NRF-2012R1A1A2003515), and the BK21 program.

\section{References}

1. Liu, G. L.; Lu, Y.; Kim, J.; Doll, J. C.; Lee, L. P. Adv. Mater. 2005, 17, 2683.

2. Shafer-Peltier, K. E.; Haynes, C. L.; Glucksberg, M. R.; Van Duyne, R. P. J. Am. Chem. Soc. 2003, 125, 588.

3. Grubisha, D. S.; Lipert, R. J.; Park, H. Y.; Driskell, J.; D. Porter, M. Anal. Chem. 2003, 75, 5936

4. Isola, N. R.; Stokes, D. L.; Vo-Dinh, T. Anal. Chem. 1998, 70, 1352.

5. Lyandres, O.; Shah, N. C.; Yonzon, C. R.; Walsh, J. T., Jr.; Gluckberg, M. R.; Van Duyne, R. P. Anal. Chem. 2005, 77, 6134.

6. Kneipp, J.; Kneipp, H.; Rice, W. L.; Kneipp, K. Anal. Chem. 2005, 77, 2381 .

7. Graham, D.; Mallinder, B. J.; Smith, W. E. Angew. Chem.-Int. Edit. 2000, 39, 1061.

8. Premasiri, W. R.; Moir, D. T.; Klempner, M. S.; Krieger, N.; Jones II, G.; Ziegler, L. D. J. Phys. Chem. B 2005, 109, 312

9. Kneipp, K.; Kneipp, H.; Deinum, G.; Itzkan, I.; Dasari, R. R.; Feld, M. S. Appl. Spectrosc. 1998, 52, 175.

10. Domke, K. F.; Zhang, D.; Pettinger, B. J. Am. Chem. Soc. 2006, 128,14721 .

11. Habuchi, S.; Cotlet, M.; Gronheid, R.; Dirix, G.; Michiels, J.; Vanderleyden, J.; De Schryver, F. C.; Hofkens, J. J. Am. Chem. Soc. 2003, 125, 8446.

12. Michaels, A. M.; Nirmal, M.; Brus, L. E. J. Am. Chem. Soc. 1999, $121,9932$.

13. Xu, H. X.; Bjerneld, E. J.; Kall, M.; Borjesson, L. Phys. Rev. Lett. 1999, 83,4357

14. Nie, S. M.; Emery, S. R. Science 1997, 275, 1102.
15. (a) Gu, G. H.; Suh, J. S. J. Phys. Chem. A 2009, 113, 8529. (b) Etchegoin, P. G.; Lacharmoise, P. D.; Le Ru, E. C. Anal. Chem. 2009, 81,682 .

16. Lee, S. J.; Morrill, A. R.; Moskovits, M. J. Am. Chem. Soc. 2006, $128,2200$.

17. Sawai, Y.; Takimoto, B.; Nabika, H.; Ajito, K.; Murakoshi, K. J. Am. Chem. Soc. 2007, 129, 1658.

18. Imura, K.; Okamoto, H.; Hossain, M. K.; Kitajima, M. Nano Lett. 2006, 6, 2173

19. Svedberg, F.; Li, Z. P.; Xu, H. X.; Kall, M. Nano Lett. 2006, 6, 2639.

20. Joo, Y.; Suh, J. S. Bull. Korean Chem. Soc. 1995, 16, 808.

21. Suh, J. S.; Lee, J. S. Chem. Phys. Lett. 1997, 281, 384.

22. Wang, H. H.; Liu, C. Y.; Wu, S. B.; Liu, N. W.; Peng, C. Y.; Chan, T. H.; Hsu, C. F.; Wang, J. K.; Wang, Y. L. Adv. Mater. 2006, 18, 491.

23. Sauer, G;; Brehm, G.; Schneider, S.; Graener, H.; Seifert, G.; Nielsch, K.; Choi, J.; Goring, P.; Gosele, U.; Miclea, P.; Wehrspohn, R. B. J. Appl. Phys. 2005, 97, 6.

24. Jeong, D. H.; Zhang, Y. X.; Moskovits, M. J. Phys. Chem. B 2004, $108,12724$.

25. Gu, G. H.; Kim, J.; Kim, L.; Suh, J. S. J. Phys. Chem. C 2007, 111, 7906.

26. Wang, D. S.; Chew, H.; Kerker, M. Appl. Opt. 1980, 19, 2256.

27. Kuwata, H.; Tamaru, H.; Esumi, K.; Miyano, K. Appl. Phys. Lett. 2003, $83,4625$.

28. Suzuki, M.; Maekita, W.; Wada, Y.; Nakajima, K.; Kimura, K.; Fukuoka, T.; Mori, Y. Appl. Phys. Lett. 2006, 88, 203121.

29. Laurent, G.; Felidj, N.; Aubard, J.; Levi, G.; Krenn, J. R.; Henau, A.; Schider, G.; Leitner, A.; Aussenergg, F. R. J. Chem. Phys. 2005, 122, 011102 .

30. Gu, G. H.; Suh, J. S. Langmuir 2008, 24, 8934

31. Masuda, H.; Hasegwa, F.; Ono, S. J. Electrochem. Soc. 1997, 144, L127.

32. Zong, R. L.; Zhou, J.; Li, Q.; Du, B.; Li, B.; Fu, M.; Qi, X. W.; Li, L. T.; Buddhudu, S. J. Phys. Chem. B 2004, 108, 16713.

33. Osawa, M.; Matsuda, N.; Yoshii, K.; Uchida, I. J. Phys. Chem. 1994, 98, 12702.

34. Lee, S. J.; Guan, Z.; Xu, H.; Moskovits, M. J. Phys. Chem. C 2007, 111, 17985

35. Qiu, T.; Zhang, W.; Lang, X.; Zhou, Y.; Cui, T.; Chu, P. K. Small 2009, 5, 2333

36. McFarland, A. D.; Young, M. A.; Dieringer, J. A.; Van Duyne, R. P. J. Phys. Chem. B 2005, 109, 11279.

37. Ruths, M. Langmuir 2003, 19, 6788.

38. Saito, Y.; Kobayashi, M.; Hiraga, D.; Fujita, K.; Kawano, S.; Smith, N. I.; Inouye, Y.; Kawara, S. J. Raman Spectrosc. 2008, 39, 1643. 